\title{
particularités du comportement mécanique des craies: rôle de l'eau rupture sous contrainte hydrostatique
}

par

\author{
R. Hazebrouck, B. Duthoit
}

Docteurs $3^{\text {e }}$ cycle, Assistants à I'I.U.T. BÉTHUNE

Liste des notations

c

: cohésion

$\varphi$

E

P

Sr

U

$\sigma_{\mathrm{rc}} \quad$ : résistance à la rupture en compression simple

$\sigma_{i j} \quad:$ contraintes totales

$\sigma_{i j} \quad:$ contraintes effectives

$\sigma_{\mathrm{g}} \quad$ : contrainte de gonflement

$\delta_{i j} \quad:$ symbole de Kronecker

$\psi$ $\left(\delta_{i j}=1\right.$ si $i=j ; \delta_{i j}=0$ si $\left.i \neq j\right)$

: frottement interne

module d'Young

pression de confinement

: humidité relative, rapport de la pression de vapeur existante à la pression de vapeur saturante à même température

\section{Introduction}

La craie blanche est réputée être un matériau évolutif sous l'effet conjugué des contraintes et de l'eau, Elle se comporte soit comme une roche soit comme un sol. II ressort des études relatives au matériau crayeux que la compréhension mécanique de la craie (Arquié, 1973, p. 8) repose sur celle de sa cohésion texturale et de ses modifications sous l'effet des contraintes et de l'eau. Par ailleurs, la dispersion des résultats des différents types d'essais sur craie est souvent importante. II y a problème quant aux limites d'utilisations des différents essais sur les craies.

A la suite de résultats acquis dans le bassin de Paris (Bull. Liaison L.P.C., 1973), nous avons fait l'analyse, sur différentes craies du Nord de la France,

- du rôle de l'eau sur des échantillons non sollicités mécaniquement puis sur échantillons sollicités sous contraintes uniaxiales :

- du comportement de craies sous contraintes isotropes, ce qui a permis d'élaborer un modèle de rupture permettant, entre autre, de déterminer le rôle du confinement dans des essais triaxiaux.

La synthèse de ces résultats nous a permis d'aborder l'étude du comportement en présence d'eau de craies sous sollicitations triaxiales.

L'évolution du matériau craie et donc les modifications texturales correspondantes étant souvent interprétées comme le résultat de variations de la pression intersititielle locale, nous avons réalisé des mesures, in situ, au coeur des échantillons testés, de la pression intersititielle locale.

\section{Présentation du matériau}

Cette étude sera limitée à la craie blanche senonienne qui peut être considérée comme la craie tendre typique du Nord de la France, des comportements identiques ont été observés sur d'autres types de craies turoniennes et cauniaciennes (Duthoit, Hazebrouck, 1976). L'observation microstructurale a été abordée, sur des surfaces de fractures métallisées, en microscopie électronique à balayage, le spectre de porosité a été défini par injection de mercure. La craie blanche (fig. 1) est formée de grains élémentaires de calcite de 0,2 à $2 \mu \mathrm{m}$ associés en amas "compacts» subsphériques de l'ordre de 3 à $15 \mu \mathrm{m}$ de diamètre. Ces amas laissent entre eux des gros pores de taille variant de 


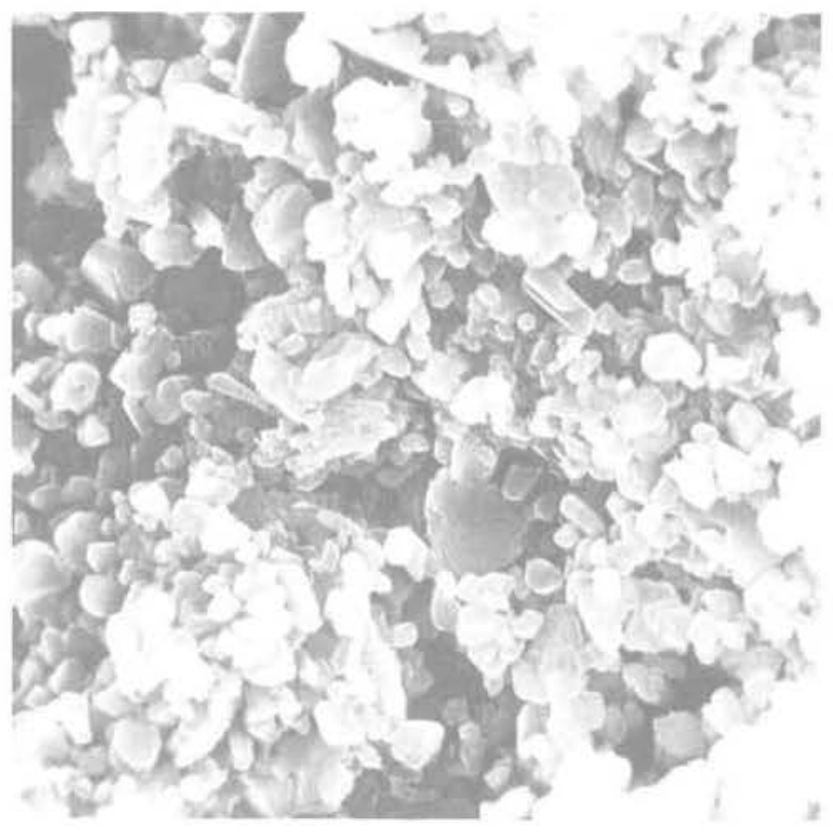

Fig. 1 Craie blanche $(3,3 \mathrm{~mm}=1 \mu)$

2 à $8 \mu \mathrm{m}$. Les vides entre particules élémentaires sont représentés par les classes de pores de rayons moyens équivalents inférieurs à $1 \mu \mathrm{m}$ avec une classe dominante entre 0,2 et $0,4 \mu \mathrm{m}$. Le Roux (1973) a montré sur des micrographies de craies le caractère restreint des liaisons entre particules élémentaires. Sans mettre en cause ce résultat que nous avons observé, nous pensons devoir pour la compréhension des déformations de la craie dissocier d'une part les liaisons entre les amas subsphériques de particules et les liaisons entre particules elles-mêmes. On peut, en première approximation, considérer la craie comme un empilement de sphères. l'empilement étant naturellement imparfait et laissant apparaître de très gros pores correspondant aux fautes d'empilement.

\section{Rôle de l'eau}

II est actuellement établi (Comès, 1973) que les craies montrent une nette diminution de la résistance à la rupture en compression simple $\sigma_{\mathrm{rc}}$ et du module d'Young $\mathrm{E}$ avec la teneur en eau. On n'a néanmoins pas, jusqu'à la présente étude, étudié systématiquement l'influence de l'humidité de l'air ou de l'eau de saturation d'un échantillon sur ces paramètres mécaniques.

Avant d'aborder l'influence de l'eau sur les propriétés mécaniques de la craie, nous examinerons le gonflement d'échantillons non soumis à un effort.

\subsection{Le gonflement des craies}

Nous avons done effectú́ les essais d'une part en chambre d'ambiance thermorégulée où l'humidité relative $\psi$ de l'air pouvait être définie et régulée et d'autre part après saturation sous vide par immersion totale des échantillons dans l'eau. Les déformations ont été mesurées en continu par capteurs inductifs (Duthoit, Hazebrouck, 1976). Ce type de manipulation devrait rendre compte des différents états hygrométriques de la craie.

Gonflement en fonction de l'humidité relative de l'air $\frac{\Delta l}{l}=f(\Psi)$

Les résultats (fig. 2) indiquent que le gonflement devient important pour une atmosphère à $45 \%$ d'humidité relative. La déformation longitudinale maximum de gonflement pour une atmosphère à $100 \%$ d'humidité à $25{ }^{\circ} \mathrm{C}$

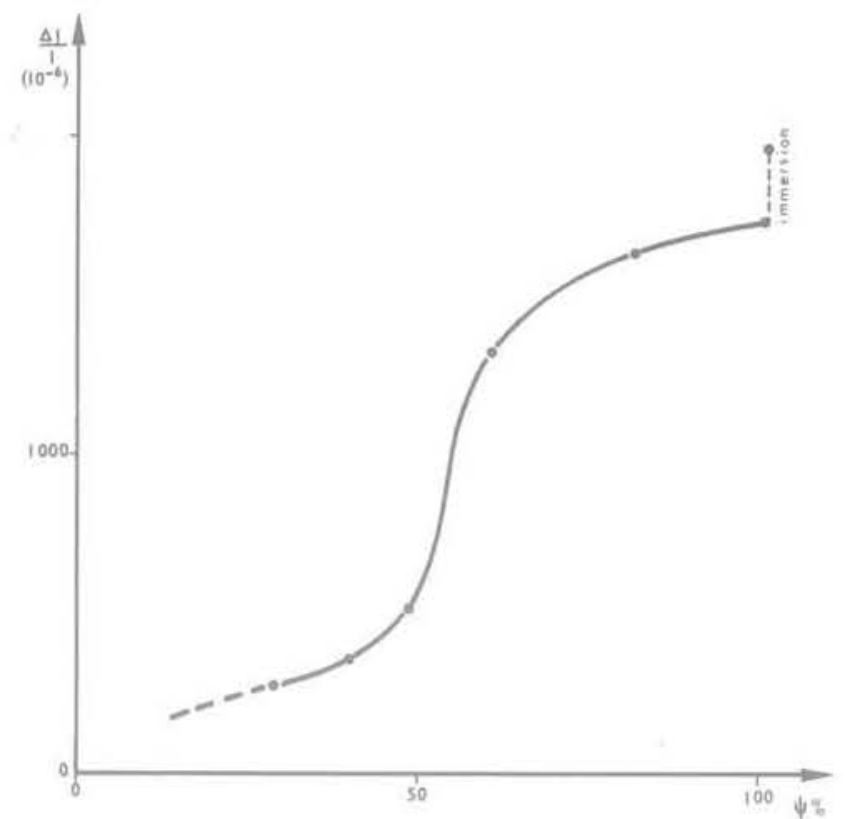

Fig. 2 Gonflement de la craie en fonction de I'humidité atmosphérique



Fig. 3 Gonflement de la craie en fonction du temps à humidité atmosphérique constante $(\psi=100 \%)$

(soit un degré de saturation de l'échantillon de l'ordre de $0,05)$, correspondrait en valeur absolue à celle obtenue en compression simple pour une contrainte moitié de celle de la résistance à la rupture.

Si l'on suppose l'isotropie du gonflement, cette craie tendre subit en atmosphère à $100 \%$ d'humidité et a fortiori à l'état saturé une déformation volumique $\frac{\Delta V}{V}$ de l'ordre de $5.10^{-3}$.

Cependant, le gonflement obtenu par immersion dans l'ethanol (faible tension superficielle) est environ deux fois plus faible que celui obtenu dans l'eau.

Gonflement à humidité et température constantes en fonction du temps $\frac{\Delta l}{l}=f(t)$

Le gonflement maximum semble acquis en 2 jours pour cette craie blanche (fig. 3), ce qui correspond environ à une vitesse de déformation $\dot{\varepsilon} \approx 210^{-8} \mathrm{~s}^{-1}$. 
Des craies à pores plus petits et à porosité plus faible présentent des vitesses et des amplitudes de gonflement moins importants (Duthoit, Hazebrouck, 1976).

\section{Interprétation}

La première conclusion ả tirer de ce gonflement des craies est que le gonflement des craies est important dès que l'atmosphère atteint $45 \%$ d'humidité après un temps relativement court de 2 jours.

Au niveau microstructural plusieurs causes peuvent expliquer ce phénomène (Powers 1968, Duthoit-Hazebrouck, 1976).

Au niveau macroscopique, on peut considérer que l'échantillon en présence d'air humide et d'eau est soumis à une contrainte isotrope de traction dite contrainte de gonflement $\sigma_{\mathrm{g}}$ (Colback et Wiid 1965, Morlier 1970).

3.2 Variations de la résistance à la rupture en compression simple $\sigma_{r c}$ et du module d'Young en fonction de l'humidité

Une cellule d'ambiance a permis de réatiser les essais en compression simple en atmosphère contrôlée ainsi qu't l'état saturé (Duthoit. Hazebrouck 1976). Afin d'assurer l'homogénéité du milieu environnant, chaque échantillon de craie a été maintenu plusieurs jours dans les conditions d'environnement de l'expérience. La figure 4 montre l'évolution de $\sigma_{r \text { r }}$ en fonction du milieu. La chute de résistance apparaît clairement pour une atmosphère dépassant $45 \%$ d'humidité, soit pour un très faible degré de saturation en eau (voisin de 0,02 ). La résistance à la rupture chute de $45 \%$ environ pour une atmosphère a $100 \%$ d'humidité $\left(S_{r}=0,05\right)$ et de $50 \%$ pour une saturation complète.

Parallèlement à cette chute de résistance une baisse du module d'Young est observée.

La chute de la résistance à la compression est directement corrélable au phénomène de gonflement examiné ci-dessus et qui se développe également à partir d'une atmosphère de $45 \%$ d'humidité. Phénoménologiquement, on peut considérer qu'une compression uniaxiale sur un échantillon de craie en atmosphère humide ou, a fortiori. s'il est saturé en eau est équivalente à celle qui s'exercerait sur un échantillon de roche sèche auquel on aurait appliqué une contrainte isotrope de traction égale à la contrainte de gonflement $\sigma_{\mathrm{g}}$ (fig. 5), ce qui explique l'abaissement de la résistance, puisque l'on ne serait plus en état de contrainte uniaxial.

Au niveau microstructural, on peut supposer une adsorption restreinte d'eau au niveau des liaisons intergranulaires entraìnant une pression locale d'écartèlement des particules (disjoining pressure) diminuant d'autant leur énergie de liaisons.

II ressort de cette étude que les conditions expérimentales d'un essai aussi simple que la compression simple doivent être strictes afin que les valeurs $\sigma_{\text {rc }}$ restent significatives et non dispersées comme on le croit fréquemment (Baguelin, 1973, p. 115).

\section{Rupture sous contrainte hydrostatique}

Au-delà d'un seuil de confinement, la craie montre un comportement de type plastique accompagné d'un tassement de sa texture (de Raguenel, 1973, p. 166). Le comportement de la craie sèche dépendra donc essentiellement de la valeur de la pression latérale. Selon Comès (1973, p. 153), au-delà de $10 \mathrm{MPa}$ de confinement, la craie deviendrait ductile et pourrait accuser des « déformations considérables \%. De telles valeurs de confinement, relativement basses, se conçoivent en tête de pieux et en ouvrages souterrains.

La microstructure particulière de la craie détermine ce type de comportement qui sera précisé en étudiant:

- le rôle de la pression isotrope de confinement sur les craies

- le comportement des craies sous composante déviatorique.

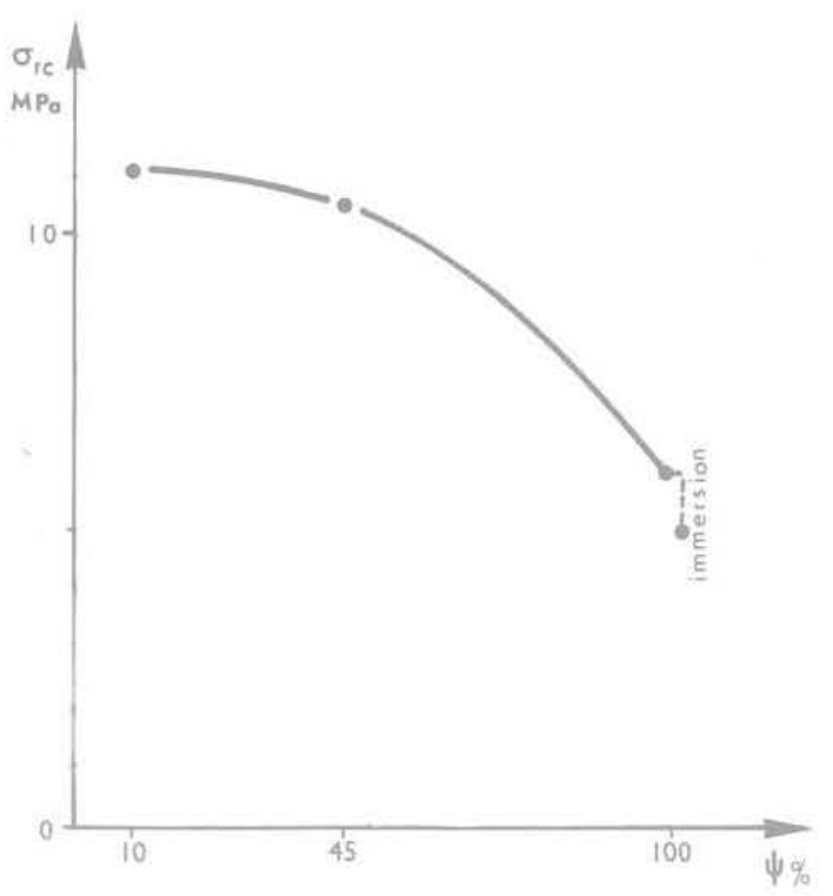

Fig. 4 Evolution de la résistance en compression simple de la craie en fonction de l'humidité atmosphérique

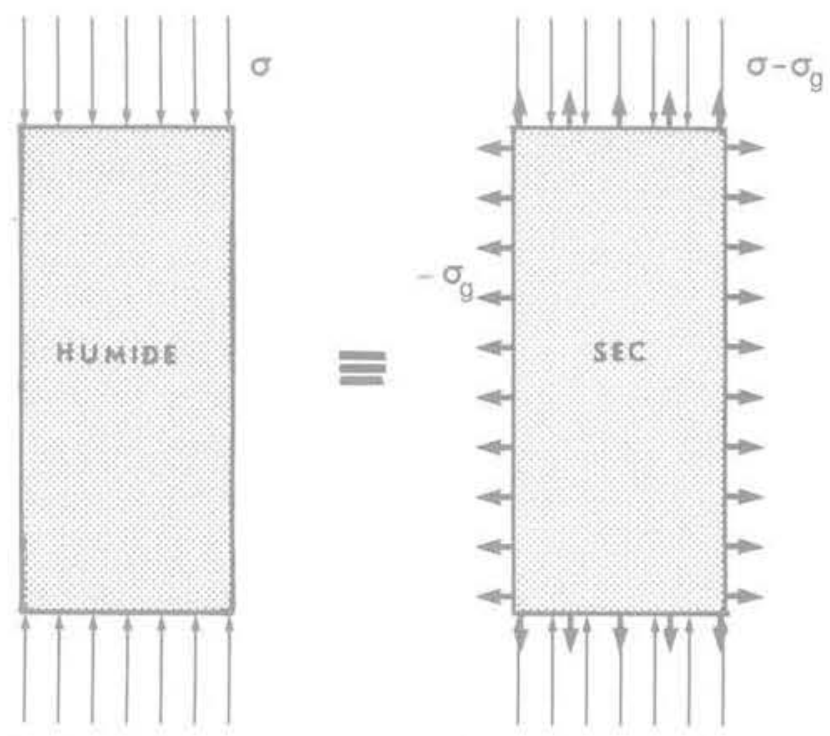

Fig. 5 Application du concept de gonflement à l'essai de compression simple

\subsection{Essais de compressibilité sur craies sèches}

Lorsqu'un échantillon de roche classique (non crayeux) est soumis à une contrainte isotrope, on observe un premier stade de déformation limitée et souvent orientée qui correspond à la fermeture de fissures aplaties du matériau (Morlier, 1970). A la suite de ce stade, on relève une absence de déformation plastique, l'échantillon présentant théoriquement, selon le principe de Bridgman, une résistance infinie sous sollicitations hydrostatiques. Les craies n'obéissent pas à ce principe et évolutent en compression isotrope. L'étude a d'abord été menée sur échantillons secs et a permis de suivre l'évolution du squelette des craies. 
Aucune anisotropie fissurale n'a été mise en évidence à la différence des roches classiques. Les déformations suivant trois directions normales ont été mesurées par jauges extensométriques collées sur les trois faces de cubes de craies : il y a linéarité des courbes contraintes-déformations dès le début de la mise en pression (fig. 6). Cette méthode doit néanmoins être abandonnée quand des déformations importantes apparaissent. Un porosimètre à mercure est alors utilisé comme cellule de confinement

jusqu'à $80 \mathrm{MPa}$. Les courbes $\mathrm{P}=\mathrm{P}\left(\frac{\Delta \mathrm{V}}{\mathrm{V}}\right)$ (fig. 7) ont été établies pour des pressions de 0 à $80 \mathrm{MPa}$, on note une première phase linéaire dès l'origine (phase A) de 0 à $15 \mathrm{MPa}$, une seconde phase de grandes déformations $\left(\frac{\Delta V}{V} \approx 10 \%\right)$ s'observe de 15 à $30 \mathrm{MPa}$ (phase $\mathrm{B}$ ), au-delà de $30 \mathrm{MPa}$, un durcissement apparaît (phase C). Après déchargement à partir de $80 \mathrm{MPa}$, l'échantillon a subi une "contraction » de l'ordre de $15 \%$ en volume.

L'évolution de la microstructure en fonction de la pression de confinement a été suivie par porosimétrie à mercure et par examen de la microstructure en microscopie.électronique à balayage, ceci pour chaque stade de la déformation.

Les courbes de porosité (fig. 8 a : $P=0, b: P=14 \mathrm{MPa}$, $c: P=25 \mathrm{MPa}, d: P=60 \mathrm{MPa}$, e $: P=80 \mathrm{MPa}$ ) montrent que lors du début de la phase $B$, les gros pores $(R>1,25$ um) disparaissent, la porosité globale varie peu (45 à $44 \%$ ). II semble donc que la perte de linéarité de la courbe $P=P\left(\frac{\Delta V}{V}\right)$ soit liée à la disparition de ces gros pores (vides inter amas).

Au cours de la phase $B$ jusqu'à $60 \mathrm{MPa}$ environ, la porosité diminue progressivement de 45 à $35 \%$ avec dérive des courbes par augmentation relative des petits pores. Durant la phase $C$ on observe une faible variation de volume et de porosité ( 35 à $34 \%$ ).

Les observations microscopiques associées à ces résultats conduisent aux conclusions suivantes:

début de la phase $A$ (fig. 9)

La structure en amas précédemment décrite est bien visible, les gros pores $(R>1,25 \mu \mathrm{m})$ correspondent aux vides inter amas.

début de la phase $B$ (fig. 10)

La structure en amas est encore visible, mais les vides inter amas ont pratiquement disparu, ceci vérifie les résultats de porosité. Il y a donc eu rupture des liaisons inter amas et réarrangement de ceux qui comblent les gros pores. En effet, l'état de contrainte au niveau de ces liaisons n'est pas hydrostatique.

\section{phase $\mathrm{C}$ au-delà de $60 \mathrm{MPa}$ (fig. 11)}

La structure en amas n'est plus visible, it $y$ a une forte densification, les liaisons intergranulaires sont pratiquement inexistantes. On se trouve donc en présence d'une poudre compactée issue de ruptures successives inter amas puis intra amas.

Par ailleurs, une décohésion plus ou moins poussée de la structure est mise en évidence dès le début de la phase B. En effet, lors d'un essai de porosité sur un échantillon prélevé à ce stade de pression, on obtient une poudre par la simple désorption du mercure.

\subsection{Essais triaxiaux (fig. 12)}

Les résultats obtenus sont concordants avec ceux de Dessenne (1971).

Pour des pressions de confinement inférieures à $10 \mathrm{MPa}$ environ, nous constatons que le déviateur de rupture croit avec la valeur de la pression de confinement. Dans la représentation de Mohr, les cercles représentatifs de la rupture admettent une droite tangente commune. Si l'on interprète cette droite en terme de frottement interne $(\varphi)$

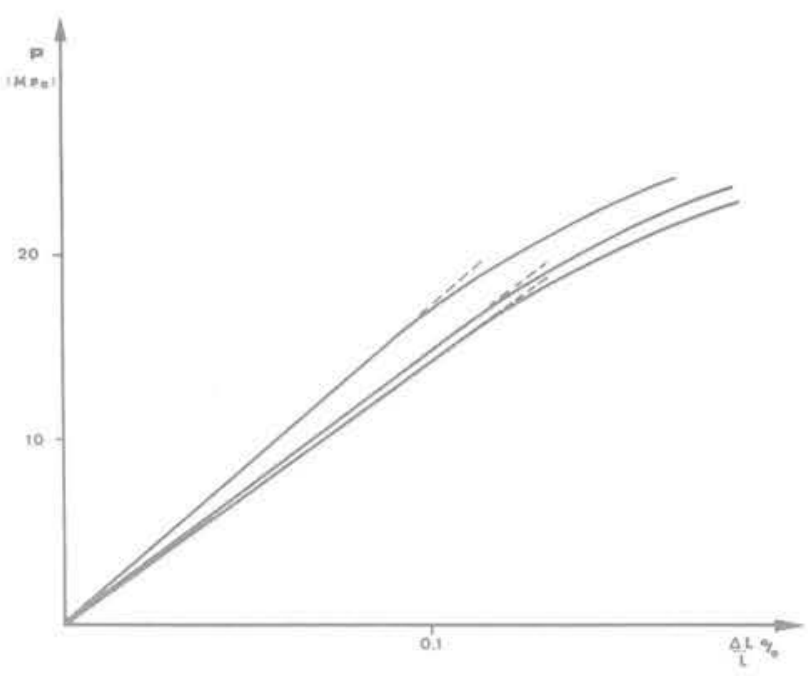

Fig. 6 Déformations longitudinales de la craie selon trois directions orthogonales



Fig. 7 Déformation volumique de la craie en essai de compressibilité

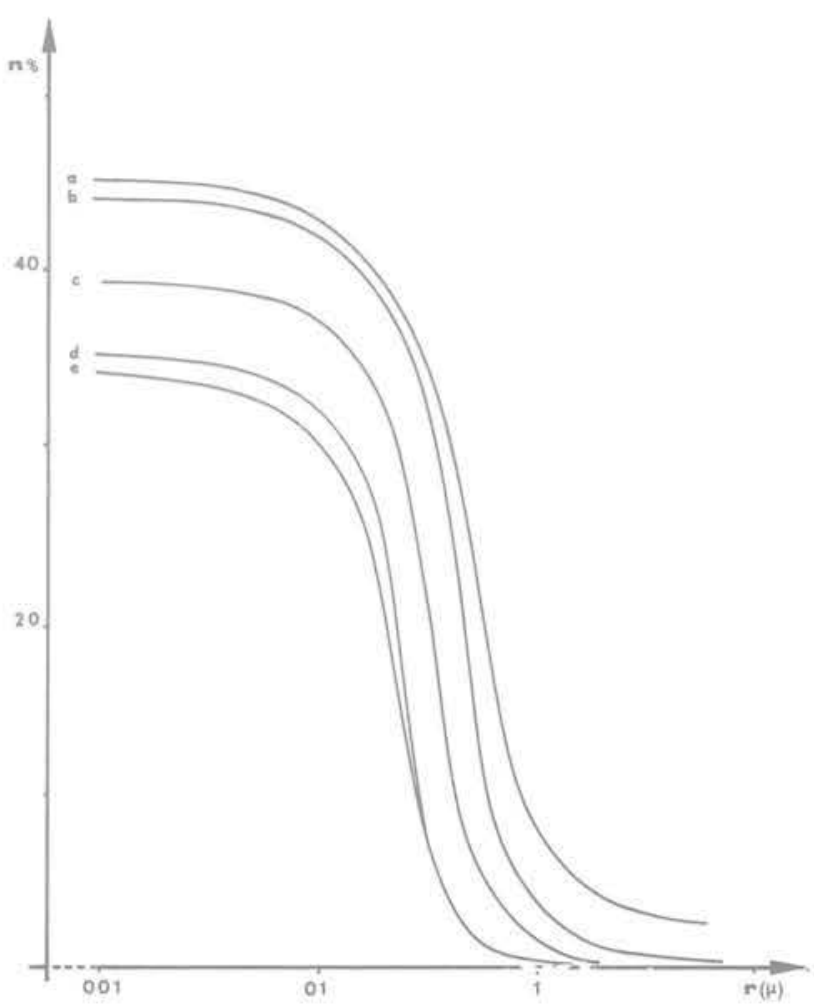

Fig. 8 Courbes cumulatives de porosité pour des échantillons prélevés à différentes pressions de confinement 


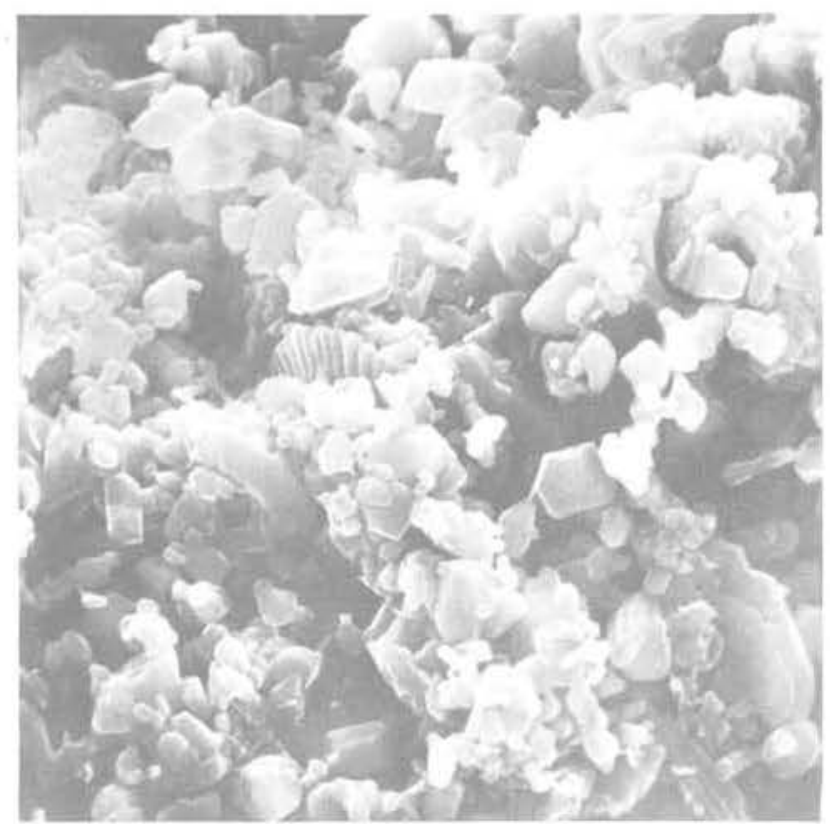

Fig. 9 Craie non déformée $(3,3 \mathrm{~mm}=1 \mu)$

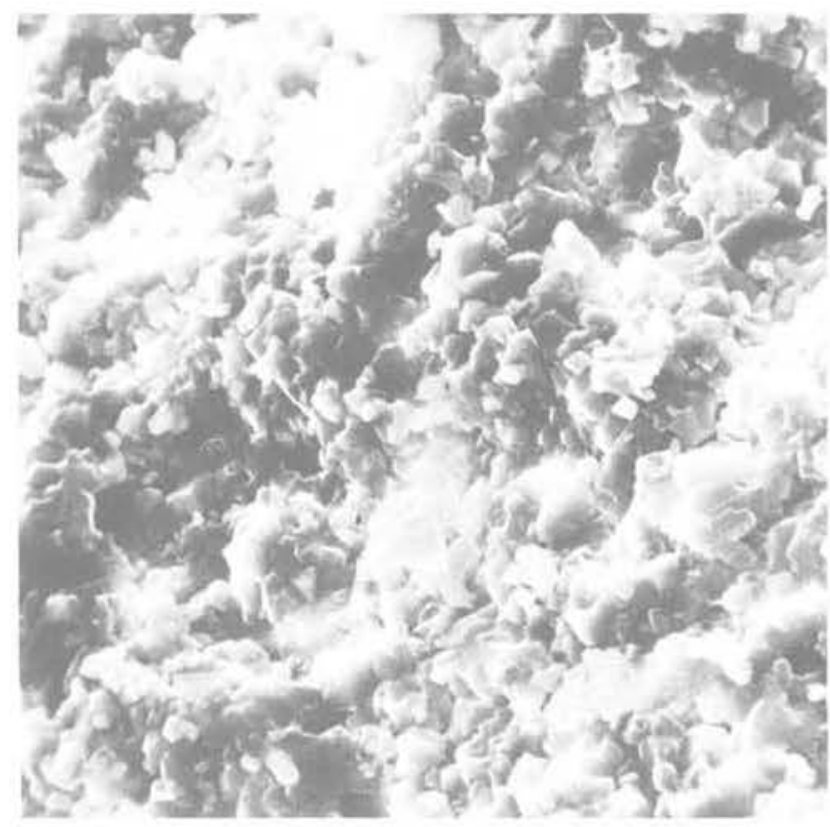

Fig. 11 Craie ayant subi une pression isotrope de $80 \mathrm{MPa}(3,3 \mathrm{~mm}=1 \mu)$



Fig. 10 Craie ayant subi une pression isotrope de $25 \mathrm{MPa}(3,3 \mathrm{~mm}=1 \mu)$



Fig. 12 Essais triaxiaux sur craie sèche

\section{Synthèse et applications}

et de cohésion (c), nous obtenons $\varphi=28^{\circ}$ et $\mathrm{c}=4 \mathrm{MPa}$. Les échantillons, après rupture, montrent des plans de cisaillement bien individualisés.

Au-delà d'une pression de confinement de $10 \mathrm{MPa}$, le déviateur de rupture décroît avec la pression latérale. Le comportement de la roche n'est plus le même, nous sommes proches de la contrainte hydrostatique ultime. La contrainte hydrostatique engendre, au niveau des liaisons inter amas, un état de contraintes proche de celui entraînant la rupture qu'un faible déviateur suffit alors à provoquer.

On observe cette fois des ruptures du type généralisé accompagnées de déformation en tonneau.

Au-delà de la contrainte ultime de confinement $l \sigma_{0} \approx 15$ $\mathrm{MPa}$, nous ne réalisons plus d'essais sur une craie roche mais sur une poudre compactée.

\subsection{Compression isotrope sur craies humides}

Pour un échantillon saturé à $70 \%$, un essai de compressibilité conduit à une courbe $\mathrm{P}=\mathrm{P}\left(\frac{\Delta \mathrm{V}}{\mathrm{V}}\right)$ (fig. 13) assez semblable au cas précédent avec relèvement à $25 \mathrm{MPa}$ environ de la contrainte à partir de laquelle il y a microrupture généralisée et densification du matériau.

Cette augmentation de résistance ne peut pas être imputée à une mise sous pression de l'eau interstitielle. En effet, des micro-capteurs de pression placés au cœur de certains échantillons (Duthoit-Hazebrouck, 1976) ont permis de vérifier que la pression interstitielle demeurait nulle lors des essais. 


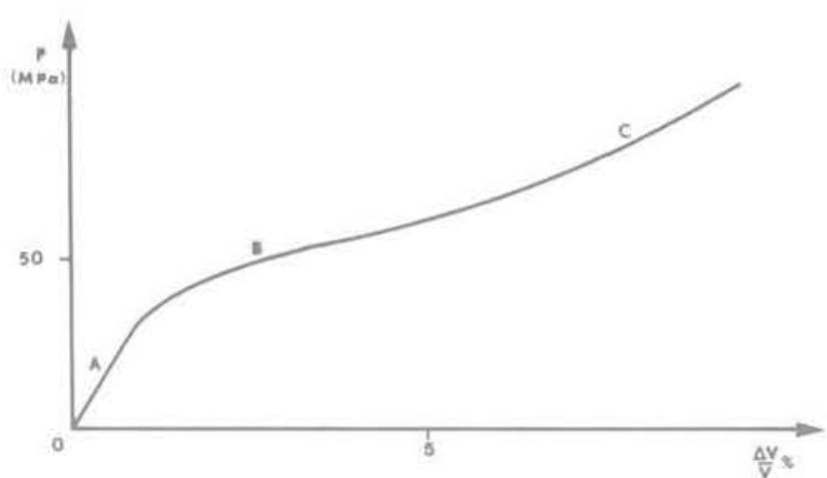

Fig. 13 Essai de compressibilité sur craie partiellement saturée $(\mathrm{Sr}=70 \%)$

Phénoménologiquement, ce comportement peut être interprété à partir du concept de contrainte de gonflement. Un tenseur de traction isotrope $\left(\sigma_{\mathrm{g}}\right)$ viendrait diminuer l'effet du tenseur de compression appliqué.

\subsection{Essais triaxiaux sur craie blanche saturée non drainée}

Les études conjuguées de la loi de variation de pression interstitielle (u) en fonction des contraintes extérieures appliquées $\left(\sigma_{i j}\right)$ et du comportement sous essais triaxiaux d'échantillons " non gainés \#, nous ont permis de formuler pour les craies étudiées, la loi de contrainte effective $\left(\sigma_{i j}{ }^{\prime}\right)$

qui comme pour un matériau pulvérulent est du type $\sigma_{i j}=\sigma_{i j}-\delta_{i j}$ u $\left(\delta_{i j}\right.$ symbole de Kronecker $)$.

Ces matériaux présentent théoriquement, pour ce type d'essai, une droite intrinsèque de rupture horizontale dans la configuration de Mohr.

La représentation des essais (fig. 14) se rapproche géométriquement de celle obtenue par Dessenne (1971). Elle montre apparemment deux comportements.

Nous interprétons le premier comportement comme le résultat d'un faible défaut de saturation des échantillons, une déformation de l'éprouvette est nécessaire pour mettre l'eau intersititielle en pression. Le second comportement suit la théorie.

\subsection{Essais triaxiaux sur craie blanche saturée} drainée

Les cercles obtenus (fig. 15) conduisent à une droite enveloppe $\varphi=27^{\circ}$ et $c=1,5$ a $2 \mathrm{MPa}$ ). Si l'on compare dans un même repère d'axes les droites enveloppes des cercles de Mohr des essais triaxiaux sur craie sèche et sur craie saturée drainée, on constate qu'elles sont à peu près parallèles et qu'elles sont superposables à une translation $\sigma_{\mathrm{g}}$ près. Le confinement serait du type $\sigma_{3}-\sigma_{\mathrm{g}}$, en retenant le rôle gonflant de l'eau qui induit, rappelons-le, une contrainte isotrope de traction $\sigma_{\mathrm{g}}$.

\section{Conclusion}

La microstructure des craies est responsable de leur comportement mécanique original.

Leur extrême sensibilité à l'eau, même en très faible quantitié, impose des conditions strictes d'essai en laboratoire. Le concept de contrainte de gonflement s'applique bien à la modélisation de leur comportement.

Sous contraintes isotropes, les craies ne vérifient pas, au niveau macroscopique, le principe de compression hydrostatique infini. Des ruptures au niveau des liaisons inter amas granulaires sont mises en évidence pour des pressions relativement faibles.

L'interprétation de résultats d'essais mécaniques sur craie devra donc se faire en tenant compte de ces deux éléments particuliers.

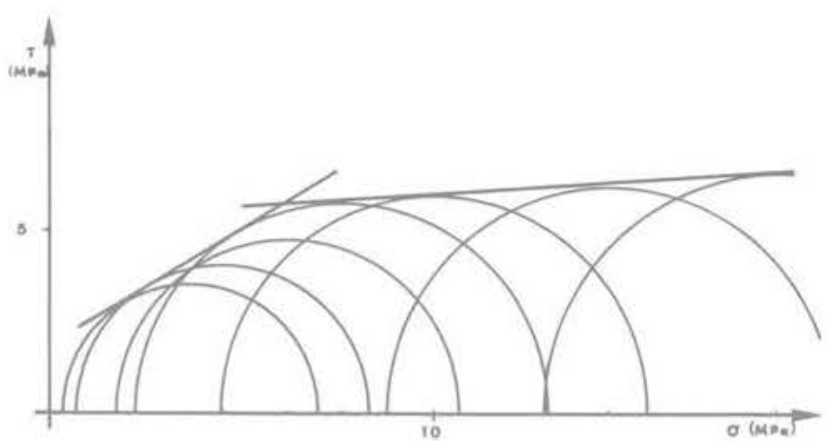

Fig. 14 Essais triaxiaux, craie saturée non drainée

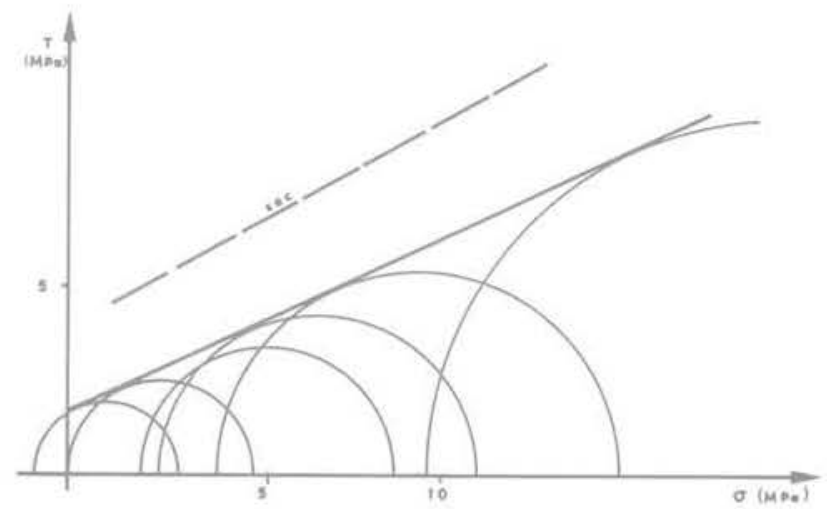

Fig. 15 Essais triaxiaux, craie saturée drainée, comparaison avec la droite intrinsèque en condition sèche

\section{Références bibliographiques}

1 - ARQUIÉ G. (1973) - Bull. Liaison L P C spécial V p. 5-8

2 - BAGUELIN F. (1973) - Fondations dans la craie - Bull. Liaison L P C spécial V p. 113-120

3 - COLBACK P.S.B. WIID B.L. (1965) - The influence of moisture content on the compressive strength of rock, Proc. Symp. Rock Mech. 3rd Toronto, p. 65-83

4 - COMÉS G. (1973) - La craie dans les ouvrages de production d'électricité, p. 149-158

5 - DE RAGUENEL A. (1973) - Bull. Liaison LP C spécial V pp. 165-169

6 - DESSENNE J.L. (1971) - Etude rhéologique et géotechnique de la craie - Thèse de docteur ingénieur - Université l de Grenoble

7 - DUTHOIT B. - HAZEBROUCK R. (1976) - Propriétés mécaniques de craies, relations avec leur microstructure, róle de l'environnement. Thèses de $3^{\circ}$ cycle - Université de Lille 1 .

8 - DUTHOIT B. - HAZEBROUCK R. - PAQUET J. (1976) Influence de l'eau sur les propriétés mécaniques de craies. Ann SGN XCVI, 1 13-20

9 - DUTHOIT B. - HAZEBROUCK R. - PAQUET J. (1977) Comportement de craies sous contraintes isotropes 10 à 800 bars). Ann. SGN XCVII, 2, 143-151

10 - LE ROUX A. (1973) - Texture et comportement des craies - Bull. Liaison spécial V - p. 49-53

11 - MORLIER P. (1970) - Comportement des roches sous contraintes en fonction de leur teneur en eau. La Houille Blanche $n^{\circ} 5$ p. $471-475$

12 - MORLIER P. (1970) - Plasticité et écrouissage d'un métal fritté - Mémoires scientifiques, Revue métallurgique, LXVII, $n^{\circ} 6$ 13 - POWERS T.C. (1968) - The thermodynamics of volume change and creep, Matériaux et construction, 1, 6, p. 487-508. 